\title{
Walter Greiner
}

ClASSICAL

ELECTRODYNAMICS

Foreword by D. Allan Bromley

With 284 Figures

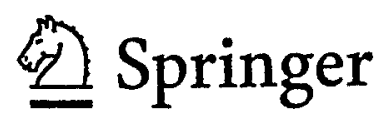




\section{Contents}

Foreword v

$\begin{array}{ll}\text { Preface } & \text { vii }\end{array}$

I Electrostatics 1

1 Introduction and Fundamental Concepts 3

2 Green's Theorems $\quad 45$

3 Orthogonal Functions and Multipole Expansion: $\begin{array}{ll}\text { Mathematical Supplement } & 70\end{array}$

4 Elementary Considerations on Function Theory: Mathematical Supplement

II Macroscopic Electrostatics $\quad 121$

5 The Field Equations for Space Filled with Matter 123

6 Simple Dielectrics and the Susceptibility 132

7 Electrostatic Energy and Forces in a Dielectric 156 
III Magnetostatics

8 Foundations of Magnetostatics

9 The Vector Potential

10 Magnetic Moment

11 The Magnetic Field in Matter

IV Electrodynamics

12 Faraday's Law of Induction

13 Maxwell's Equations

14 Quasi-Stationary Currents and Current Circuits

15 Electromagnetic Waves in Vacuum

16 Electromagnetic Waves in Matter

17 Index of Reflection and Refraction

18 Wave Guides and Resonant Cavities

19 Light Waves

20 Moving Charges in Vacuum

21 The Hertzian Dipole

22 Covariant Formulation of Electrodynamics

23 Relativistic-Covariant Lagrangian Formalism

24 Systems of Units in Electrodynamics: Supplement

25 About the History of Electrodynamics 